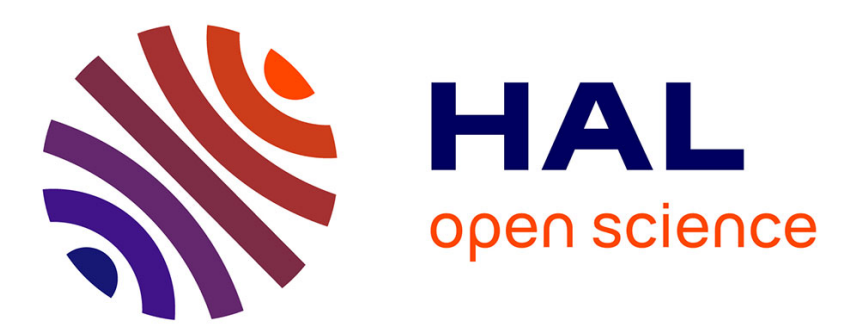

\title{
"Fragments inédits de la Physica : contribution à l'étude de la transmission des manuscrits scientifiques de Hildegarde de Bingen"
}

Laurence Moulinier

\section{- To cite this version:}

Laurence Moulinier. "Fragments inédits de la Physica: contribution à l'étude de la transmission des manuscrits scientifiques de Hildegarde de Bingen". Mélanges de l'École française de Rome - Italie et Méditerranée, 1993, 105, fasc. 2, pp.629-650. halshs-00608746

\section{HAL Id: halshs-00608746 \\ https://shs.hal.science/halshs-00608746}

Submitted on 23 Sep 2011

HAL is a multi-disciplinary open access archive for the deposit and dissemination of scientific research documents, whether they are published or not. The documents may come from teaching and research institutions in France or abroad, or from public or private research centers.
L'archive ouverte pluridisciplinaire HAL, est destinée au dépôt et à la diffusion de documents scientifiques de niveau recherche, publiés ou non, émanant des établissements d'enseignement et de recherche français ou étrangers, des laboratoires publics ou privés. 
Deux fragments inédits de la Physica :

contribution à l'étude de la transmission des manuscrits scientifiques de Hildegarde de Bingen

L'encyclopédie naturelle composée vers 1150 par sainte Hildegarde de Bingen (1098-1179) et connue aujourd'hui sous le titre de Physica a été éditée au siècle dernier par Charles-Victor Daremberg dans la Patrologie latine d'après l'actuel ms lat. 6952 de la Bibliothèque Nationale'1. Outre cette version du XVe siècle, la Physica nous a été conservée dans quatre autres manuscrits complets : le Cod. Guelf. 56, 2. Aug. 40 de la Herzog August Bibliothek de Wolfenbüttel (début XIVe siècle), le Cod. 2551 de la Bibliothèque Royale de Bruxelles (XVe ou XVle siècle selon les auteurs ${ }^{2}$ ) et deux manuscrits récemment mis au jour. Le ms. Ashburnham 1323 de la Biblioteca Laurenziana de Florence (vers 1300) a été découvert en 1983 par Paulus Becker, et le Ferraioli 921 de la Biblioteca Apostolica Vaticana (XVe siècle) en 1985 par Ursula Heierle.

A cela s'ajoutent quelques fragments, du XVe siècle pour la plupart, contenus dans les manuscrits suivants : le Cod. 525 de la Burger Bibliothek de Berne, le manuscrit 178a de I'Universitätsbibliothek de Fribourg-en-Brisgau (découvert et édité par Raimund Struck en 1985³) et le Cod. III 1, fol. 43 de la Bibliothèque Öttingen-Wallerstein de Harburg, aujourd'hui à Munich, Bayerische Staatsbibliothek, auquel Melitta Weiss-Amer vient de consacrer un article ${ }^{4}$. Ce dernier fragment a pour particularité son bilinguisme, et des passages de la Physica en latin s'y mêlent à d'autres dans leur traduction allemande ; un autre indice de la transmission de cette œuvre en langue vernaculaire nous est

1Sanctae Hildegardis abbatissae subtilitatum diversarum naturarum creaturarum libri novem (Physica), dans Patrologiae cursus completus, series latina, tome 197 (désormais cité PL 197), éd. J.-P. Migne, Paris, 1855, c. 11171352.

2Vers 1440 selon Roger Calcoen (Inventaire des manuscrits scientifiques de la Bibliothèque Royale de Belgique, 1965, tome 1, p. 52), XVle siècle d'après Willem Lourdaux et Marcel Haverals (Bibliotheca Vallis Sancti Martini in Lovanio. Bijdrage tot de Studie van het geestleven van de Nederlanden [15de18de eeuw], Louvain, 1978, p. 465).

${ }^{3}$ Cf. Raimund Struck, Hildegardis De Lapidibus Ex Libro Simplicis Medicinae, Kritische Edition unter Vergleich anderer Lapidarien, Med. Diss., Marburg, 1985.

${ }^{4}$ Cf. M. Weiss-Amer, "Die ,Physica' Hildegards von Bingen als Quelle für das ,Kochbuch Meister Eberhards"', Sudhoffs Archiv, 76, Heft 1, 1992, pp. 87-96. 
d'ailleurs donné par le ms. Germ. Fol. 817 de la Preussische Staatsbibliothek

de Berlin : ce manuscrit inédit, que Jessen signalait en $1864^{5}$, contient en effet une traduction allemande de la praefatio du livre premier de la Physica.

Or c'est avec quatre nouveaux fragments en latin qu'il faut désormais compter : I'un d'entre eux a été signalé le premier par Ludwig Schuba mais est toujours inédit (ms. Pal. lat. 1207, f. 65v), et une bourse d'études de l'Ecole Française de Rome m'a permis d'en découvrir un second dans le même manuscrit (f. 64r), ainsi que deux fragments anonymes à l'intérieur de recueils faisant également partie du fonds Palatin latin de la Bibliothèque Vaticane. Ce sont ces deux fragments anonymes (ms. Pal. lat. 1216, ff. 91v-95r, et ms. Pal. lat. 1144, ff. 128v-129r), dont nous donnons une transcription en annexe, qui nous retiendront plus longuement ici.

On connaît bien aujourd'hui I'histoire de la bibliothèque palatine, cédée au pape Grégoire XV par le duc de Bavière Maximilien ler après la prise de Heidelberg par Tilly en 1622, et acheminée vers Rome en 1623 sous la surveillance de Leone Allacci6. Cette riche bibliothèque, dont l'origine remonte au prince électeur Ruprecht ler, fondateur de l'université de Heidelberg en 1386, rassemblait, outre la bibliothèque palatine proprement dite, plusieurs collections conservées en divers endroits (la bibliothèque de la faculté des arts, celles des autres facultés et celle de l'église du Saint-Esprit) et elle n'avait cessé de s'enrichir au cours des XVe-XVle siècles grâce aux achats de bibliothèques, aux legs de professeurs de l'université ou aux dons des électeurs.

\section{Le ms. Pal. lat. 1216 (cf. Annexe A)}

Le ms. Pal.. lat. 1216 est un recueil, latin-allemand, ayant appartenu au comte palatin Ottheinrich (1502-1559), "grand amateur de manuscrits et de livres"7, qui le fit relier en 1556 (une gravure le représente sur la reliure, avec les initiales $\mathrm{O}[\mathrm{tt}] \mathrm{H}[$ einrich] $\mathrm{C}$ [omes] $\mathrm{P}$ [alatinus]). Ce recueil de 268 folios sur

${ }^{5}$ Cf. Carl Jessen, Botanik der Gegenwart, Leipzig, 1864, p. 123. Pour une description de ce manuscrit, voir Hermann Degering, Kurzes Verzeichnis der Germanischen Handschriften der preussischen Staatsbibliothek, Leipzig, 1925, p. 114.

6Voir entre autres la notice que lui consacre Colette Jeudy ("Fonds palatin") dans Les manuscrits classiques latins de la Bibliothèque Vaticane, catalogue établi par Elisabeth Pellegrin, Paris, CNRS, t. II, 2e partie, 1982, pp. 9-17. ${ }^{7}$ C. Jeudy, "Fonds palatin", loc. cit., p. 12. 
papier de $21 \times 15 \mathrm{~cm}$, contient des extraits, copiés à différentes époques,

d'auteurs tels Vindicianus, Cophon" ${ }^{8}$, le Pseudo-Hippocrate, Jean de Parme, Arnaud de Villeneuve, Jean de Roquetaillade, etc. - une inscription du XVle siècle au folio ar indique laconiquement : "Experimenta ad varios morbos collecta ex diversis authoribus". La première partie de ce recueil (ff.1-107) date, selon L. Schuba ${ }^{9}$, de la fin du XIVe, avec des ajouts du XVe siècle; la seconde (ff. 108-260, les derniers folios étant vierges) daterait quant à elle des années 1445-1480 et proviendrait de la région de Worms (cf. f. 224r : ...datum per magistrum montani de Wormacia qui habuit illud a domicello de Lutzenburg).

Auparavant, du milieu à la fin du XVe siècle, il fut la propriété de Nicolaus Hasel, curé de Pfiffligheim près de Worms, puis de Johannes Ceci : ...finitum anno domini $1445^{\circ}$ in die Nazarii per manum Nicolai Hasel plebani in Peffelkeim, f. 97r ; ...anno domini 1480 pridie Margarethe virginis Nicolaus Hasel subscripsit, f. 252v, et ...qui scripsi expertus sum LXXXIIII (1484) Johannes Ceci, f. 78r.

C'est toutefois une main inconnue par ailleurs dans le reste du recueil qui a copié les folios $91 \mathrm{v}$ à $95 \mathrm{r}$, à l'encre marron (souvent bue par le papier), sur une seule colonne ; le folio $90 \mathrm{v}$ est blanc, le folio $95 \mathrm{v}$ ne porte que des traces d'essais de plumes (In nomine domini Amen ..), et l'on a trace d'un feuillet coupé entre les ff. 90 et 91 . Les folios 91 à 95 se présentent donc comme un ensemble cohérent et isolé, objet de paginations successives : une numérotation de 91 à 95 , en chiffres arabes, probablement du XVle siècle; une autre, antérieure, en chiffres romains, de LXXXXII à LXXXXVI. Elle-même est née en fait de modifications apportées à l'encre à une foliotation précédente, également en chiffres romains, de LXXXIX à XCIII.

Deux mains se succèdent dans cette série : le recto du folio 91 est occupé pour sa part par huit remèdes à base de vin ${ }^{10}$, tandis que c'est sur des remèdes pour les animaux domestiques (Si boves aut oves aliquid mali...) que s'ouvre le verso du même folio, d'une écriture plus grosse. Cette seconde main a copié les folios $91 \mathrm{v}-95 \mathrm{r}$, couverts d'une trentaine de lignes en moyenne ${ }^{11}$ : ils

\footnotetext{
${ }^{8}$ Auteur auquel est attribuée une Anatomie du porc datant des années 10801090.

${ }^{9}$ Pour une description complète du recueil voir L. Schuba, Die medizinischen Handschriften der Codices Palatini Latini in der Vatikanischen Bibliothek, op. cit., pp. 210-218.

${ }^{10}$ Inc. : Vinum gramineum fit de radicibus....Des.: Vinum nobilissimum quod stringit fluxum et confortat fit de suco aconitorum et depurato cum vino et aqua rosata

11F. $91 v$ : 33 lignes, f. 92r : 31 I., f 92v : 34, f. 93r : 32, f. 93v : 29, f. 94r : 29, f. 94v : 29, f. 95r : 20.I.
} 
reproduisent 42 recettes médicales, et ces recettes ne sont autre que des extraits de différents chapitres de la Physica. Un même chapitre a pu fournir plus d'une recette, et l'on reconnaît ici en tout 37 chapitres répartis entre 6 des 9 livres qui constituent la Physica : 23 chapitres du livre premier, de plantis, 4 du livre III, de arboribus, 5 du livre IV, de lapidibus, un du livre V, de piscibus, 3 du livre VI, de avibus, et un du livre IX de metallis.

A la lecture de ce fragment, une première remarque s'impose, concernant l'ordre de succession des recettes : il ne reflète en effet pas, comme la table de concordances permet de s'en rendre compte (Annexe C), l'ordre de succession des différents livres de la Physica, ni même, à l'intérieur de chaque livre, l'ordre dans lequel les chapitres se suivent. Les recettes à base de pierres précieuses (38 à 42) font certes exception, puisqu'elles sont toutes regroupées à la fin de notre fragment, et énumérées dans l'ordre qui est celui du livre IV de la Physica : la médecine minérale est présentée ici comme à part, et introduite par quelques lignes qui la justifient ${ }^{12}$, et il faut ici se souvenir que le "livre des pierres" a pu être copié séparément, comme le prouve l'actuel fragment de Fribourg ${ }^{13}$. La phrase Cum non sit dubium...iam propono scribere ne figure en effet pas dans la Physica : le scribe prend ici la parole, tout autant que l'initiative. Mais l'ordre dans lequel sont cités tous les autres chapitres est le produit d'une réorganisation, et notre fragment introduit de nouvelles entrées, une nouvelle grille de lecture. Alors que la Physica se présentait comme un "livre de simple médecine" (sous-titre qui lui fut donné dès le début du XIlle siècle) passant en revue les créatures des différents règnes en autant de chapitres énumérant systématiquement leurs vertus médicinales, le ms. Pal. lat. 1216 lui substitue une médecine composée opérant un certain nombre de regroupements. La transcription se double donc ici d'un réagencement.

Vient ainsi en tête un groupe de dix recettes de médecine vétérinaire, où sont d'abord envisagées les maladies du bétail, puis deux relatives aux chiens où prime en fait le souci de l'homme (comment calmer un chien enragé, et donc s'en préserver [A9], et que faire en cas de morsure [A10] ?). Suivent deux

\footnotetext{
${ }^{12}$ Sur le rôle joué par les seules pierres précieuses chez Hildegarde, on peut se reporter entre autres à Peter Riethe, Hildegard von Bingen, Das Buch von den Steinen, Salzbourg, O. Müller Verlag, 1979.

${ }^{13}$ Fribourg, Universitätsbibliothek, ms. 178a, ff. 1-15r. Pour une description de ce manuscrit, voir Winfried Hagenmaier, Kataloge der Universitätsbibliothek Freiburg im Breisgau, Bd. 1, Teil 1, Die lateinischen mittelalterlichen Handschriften der Universitätsbibliothek (Hs. 1-230), Wiesbaden, Otto Hanassowitz, 1974, p. 169.
} 
recettes contre bubons et pustules, trois autres contre divers parasites ("vers" des jardins, vers de l'homme et du bétail, et mouches), puis quatorze recettes contre différentes maladies de l'homme - apparemment énumérées selon l'ordre a capite ad calcem si l'on excepte un remède contre le mal d'estomac (A19) qui vient s'immiscer parmi les maux de la tête (surdité, ophtalmies, etc.), entre une médication contre la "voix rauque" et deux recettes contre le "cerveau vide".

Les recettes 31 à 34 rompent pour leur part avec l'entrée par maladie au profit du type de préparation médicale, et l'on voit alors se succéder un onguent, une "bonne poudre", une "excellente poudre" et un électuaire. Notons à ce sujet d'une part que la médecine de Hildegarde se fait tout autant curative que préventive, et d'autre part qu'une hiérarchie de ces préparations médicales est esquissée, qui fait de certaines d'entre elles une véritable panacée : de même que "I'herbe appelée peffircrut" était présentée comme "bonne et utile pour les malades comme pour les bien-portants" (A30), la "bonne poudre" (A32) convient également aux hommes sains et aux malades, qu'elle maintient en bonne santé ou ramène à cet état, en leur assurant la même "bonne mine". L'excellente poudre"(A33) est elle aussi recommandée à ces deux catégories, mais elle surpasse la précédente, et mérite donc son qualificatif, dans la mesure où elle est gage d'une bonne santé durable. L'électuaire à base de poires (A34) est sans doute toutefois plus louable encore, et qualifié à ce titre de "plus utile et plus précieux que l'or", dans la mesure où "il débarrasse I'homme de toutes ses humeurs mauvaises comme on nettoie un vase de ses excréments".

Leur commune efficacité contre différents types de "fièvres" (quartes, tierces, quotidiennes) motive ensuite la succession des recettes 35 à 37 , et le fragment s'achève, on l'a dit, sur une série de 5 recettes à base de pierres dans laquelle on peut distinguer un ultime regroupement : après la vertu de l'émeraude contre "les vers qui mangent l'homme" sont énoncées en effet celles que possèdent hyacinthe, onyx, saphir et topaze (A39 à 42) dans une même perspective, les affections oculaires. C'est ici le principe médical similia similibus curare qui commande l'emploi de ces pierres : elles ont en effet de fortes affinités avec les yeux selon Hildegarde, qui les tient pour également composés d'eau et de feu ${ }^{14}$.

${ }^{14}$ Comparer par exemple le début du "livre des pierres" (Omnis lapis ignem et humiditatem in se habet, Physica, PL 197, c. 1247C) à ce qui est dit des yeux dans le Causae et curae: Oculus autem ex igne et aqua constat. Igne enim continetur et roboratur, ut sit; aqua vero ad visum ducitur. (Beate Hildegardis Causae et curae, éd. P. Kaiser, Leipzig, Teubner, 1903, p. 92). 
Ce fragment est également remarquable d'un point de vue strictement linguistique, car il est parsemé de termes vernaculaires. La lecture du texte permet de se rendre compte que le copiste connaissait le latin sans en maîtriser parfaitement la grammaire, mais on ne saurait pour autant lui imputer de manière certaine le recours à un vocabulaire germanique qui figurait déjà peut-être dans le manuscrit qu'il démarquait : le latin n'était pas non plus la langue maternelle de Hildegarde et tous les manuscrits complets de la Physica connus à ce jour contiennent des termes en langue vulgaire. Ces différentes versions étant nettement postérieures à la date de composition de l'œuvre originale, on peut certes y voir l'intervention de copistes ; toutefois il est très probable que l'abbesse elle-même ait employé ces termes, faute de connaître par exemple le nom latin de toutes les plantes indigènes qu'elle avait entrepris de décrire.

De fait c'est dans le domaine botanique que les emprunts à la langue vulgaire sont ici les plus nombreux : 13 noms de végétaux sont traduits, alors que l'équivalent vernaculaire n'est donné que pour trois noms de maladies ("constrictio guthuris id est strengelen" et "dolor capitis id est heubtsucht"[A8], "bubo id est sler" [A11]), d'un verbe ("si pabulum nimis avide sumpsit, quod volgariter dicitur virfangen vel zerese"[A4]) et de deux noms d'insectes ("eruce id est rupen et papiliones id est cyfelder" [A13]) $)^{15}$.

Il en va diversement des arbres ou des plantes : le nom latin d'une espèce peut être accompagné de synonymes eux-mêmes latins comme pour le Calendula ("Ryngele sive flammula" [A1]) et l'Arum ("herba que dicitur aaron id est jarus vel serpentina" [A12] $)^{16}$, ou être un simple calque linguistique de son nom vernaculaire ("lingua cervina" [A24] ne désigne pas la "langue du cerf" mais est la traduction littérale de Hirtzunge, "Hirschzunge", Scolopendre ${ }^{17}$ ) ; il

15"Rupe" a donné l'allemand actuel "Raupe","chenille", et "Falter", "papillon", est aujourd'hui le descendant du vieil haut-allemand vivaltra, du germanique *fifaldron.

16D'après Hermann Fischer, cette plante était également appelée jarus et serpentaria minor dans le Gart der Gesundheit, version allemande du Hortus sanitatis (cf. H. Fischer, Mittelalterliche Pflanzenkunde, Munich, 1929, p. 260) ; Jerry Stannard relève pour sa part serpentaria dans une version du XIIle siècle du Circa instans de Matthaeus Platearius.(J. Stannard, "Identification of the plants described by Albertus Magnus, De vegetabilibus lib. VI"', Respublica Litterarum, II, 1979, pp. 231-318, p. 288. s. v. basilicus). Le nom d"'Aaron" n'est là pour sa part que par déformation du latin Arum (cf. G. Hegi, H. Marzell, Illustrierte Flora von Mitteleuropa, Munich, 1906, vol. II, p. 132).

${ }^{17}$ Sur l'importance des noms d'animaux dans la formation des noms de plantes en allemand, on consultera Heinrich Marzell, Die Tiere in deutschen Pflanzennamen, Heidelberg, 1913. Le nom de "langue de cerf" ou "herbe à la 
peut également être suivi, et c'est le cas le plus fréquent, du nom vulgaire lui correspondant exactement, comme dans les glossaires latins-allemands que les recueils scientifiques d'origine germanique comportent la plupart du temps ${ }^{18}$ : "esculus id est spirbaum" (Sorbier) [A13] , "zizaniam id est rade" (Cumin noir ou Nigelle cultivée) [A15], "semperviva id est huswurtz" (Joubarbe) [A17], "pentafilon funfblat" (Potentille) [A23], "solatrum id est nachtschade" (Morelle noire) [A24], "tanacetum id est reynevane" (Tanaisie) [A26], "rustica id est byverwurz" (Aristoloche) [A33]. Enfin, le nom latin peut manquer purement et simplement dans le cas du Lierre ("herba que dicitur gunderebe" [A16]), de I'Epurge ("herba que brachwurtz dicitur" [A18]), de la Sariette ? ("herba que dicitur peffircrut" [A29]) ${ }^{19}$ et de l'Athamante ("herba que dicitur berwurz" [A33]).

Notons pour finir que ce fragment s'écarte, en quelques endroits, du texte de la Physica tel que nous le connaissons : la mention et pulverem piretri et cuperrose id est nesewurz et cuperrauch simul commisce, postea pulverem calido vino adjunge [A15], où interviennent l'ellébore et le vitriol20, constitue un ajout par rapport au chapitre "De Ratde" reproduit par Migne21; de même, l'indication vel hyrcini [A31], qui propose d'employer de la graisse de bouc à la place de la graisse de cerf selon un principe de substitution à l'œuvre dans d'autres recettes ${ }^{22}$, ne figure pas dans le chapitre "De unguento Hilarii"23. La

rate" est également attesté pour cette plante dans l'aire francophone; cf. G. Hegi, H. Marzell, Illustrierte Flora von Mitteleuropa, Munich, 1906, vol. I, p. 34. 18 Un tel glossaire se trouve dans ce manuscrit aux folios 155r-171v.

${ }^{19}$ Aujourd'hui encore, le terme de peffercrut reste difficile à traduire : Pierre Monat le rend par "Cresson" (Hildegarde de Bingen, Le livre des subtilités des créatures divines (Physique), trad. P. Monat, Grenoble, Jérôme Millon, 198889, 2 vol., vol. 1, p. 64), Danielle Delley lui préfère "Sariette", Satureia montana (D.Delley, Hildegarde de Bingen et les plantes médicinales, Bâle, BHG, 1988, p. 58), et Marie-Louise Portmann le traduit en allemand par "Pfefferkraut" (Hildegard von Bingen, Heilkraft der Natur 'Physica', Augsburg, Pattloch Verlag, 1991, p. 70), strict synonyme de "Bohnenkraut", "Satureja" selon le Wahrig (cf. Gerhard Wahrig, Deutsches Wörterbuch, Munic, 1986, p. 287 s. v. "Bohenenkraut").

${ }^{20 " N i e s w u r z " ~ e s t ~ l e ~ n o m ~ g e r m a n i q u e ~ d e ~ l ' e l l e ́ b o r e ~ n o i r e, ~ e t ~ " K u p f e r r a u c h " ~ o u ~}$ "Kupferrose" pour "vitriol" est déjà fréquent dans le vocabulaire du XVe siècle ; cf. Grimm, Deutsches Wörterbuch, tome V, Leipzig, 1873, p. 2767.

${ }^{21}$ Cf. Physica, I, 12, PL 197, c. 1133D : Sed et ratden tunde et mel ei commisce ; et ubi multae muscae sunt, in parietem linias, id est striche (...).

${ }^{22}$ Voir dans notre seul fragment la recette [A15], qui propose d'employer du lait si l'on n'a pas de miel, et la recette [A34] qui invite à substituer des racines de fenouil au "peucedanum" si celui-ci fait défaut.

${ }^{23}$ Cf. Physica, III, 58, PL 197, c. 1246A : ...postea laurinum oleum accipe, et bis tantum cervini sepi, et ad ejus tertiam partem veterem arvinam... 
posologie n'est pas la même dans la recette 34, "Electuarium de piris" et dans

le chapitre "De Birbaum" auquel elle est empruntée ${ }^{24}$; la recette 35 constitue pour sa part un raccourci fort éloigné de son original, le chapitre "De

plantagine"25; enfin la recette 33 présente une conclusion très abrégée de celle du chapitre "De Byverwurtz"26.

La pharmacopée végétale dans ce fragment est donc dominante mais non exclusive, et les médications à base de végétaux (plantes le plus souvent, mais aussi arbres) côtoient ici des recettes utilisant arêtes de brochet, tête d'alouette, vessie de paon ${ }^{27}$, ou encore cuivre et pierres précieuses, c'est-à-dire tirées de l'ensemble des livres de la Physica. C'est en revanche du seul "livre des plantes" qu'un autre fragment anonyme, copié aux folios $128 \mathrm{v}-129 \mathrm{r}$ du ms. Pal. lat. 1144, nous livre quelques extraits.

L'actuel fonds palatin latin de la Bibliothèque Vaticane contient, on l'a dit, un grand nombre de manuscrits scientifiques. Ludwig Schuba a dressé le catalogue de ces manuscrits médicaux et son travail nous a ainsi appris qu'une Summa Hildegardis de infirmitatum causis et curis se trouvait à Heidelberg au début du XVe siècle : un tel titre est en effet mentionné parmi les livres légués par le prince électeur Ludwig III (†1436) à l'église du Saint-Esprit, et dont le recteur de I'Université, Johannes Rybeisen von Bruchsal, dressa une liste en $1438^{28}$.

Le testament de Ludwig III prévoyait qu'après sa mort ces livres seraient conservés sous bonne garde à l'église du Saint-Esprit, et mis à la disposition des différentes facultés ; quand le recteur de l'Université en accuse bonne

${ }^{24}$ Cf. Physica, III, 2, PL 197, c. 1219A : ...et cotidie quantum cocleare unum parvum capit, jejunus comede, et pransus quantum duo coclearia, et ad noctem in lecto quantum tria coclearia.(...).

${ }^{25}$ Elle ne paraît pouvoir être qu'un lointain dérivé de cette recette contre le gicht : Accipe ergo plantaginem et succum ejus exprime, et per pannum colatum aut vino aut melle tempera, et da illi bibere qui a gicht fatigatur, et gicht cessabit. (Physica, I, 101, PL 197, c. 1169BC).

${ }^{26}$ Cf. Physica, I, 146, PL 197, c. 1189A : Sed pulverem per pannum servare poteris sanum, eum in novum fictile vas pone de limo factum et aridum, et ita sub terra funde, et clausum tege, atque sic vires obtinebis.

${ }^{27}$ Le terme de vesica figure dans les autres version de la Physica, mais pas dans la recette A11, pourtant clairement empruntée au 3e chapitre du "livre des oiseaux", "De pavone"; le scribe emploie ici le terme très générique destruma, "tumeur".

${ }^{28} \mathrm{Cf}$. L. Schuba, Die medizinischen Handschriften der Codices Palatini Latini in der Vatikanischen Bibliothek, Wiesbaden, 1981, pp. XXI, XXVI et XXVII (Katalog der Universität Heidelberg, Band I). 
réception en 1438, il range donc les manuscrits par discipline ; pour la médecine, il prend soin de séparer les ouvrages sur papier des manuscrits de parchemin, et indique le début des œuvres ${ }^{29}$. On reconnaît ainsi dans la Summa Hildegardis de infirmitatum causis et curis le second volet de l'œuvre scientifique de Hildegarde, le traité Causae et curae, que nous a transmis un unique manuscrit du XIIle siècle ${ }^{30}$. Le manuscrit attesté par Johannes Rybeisen von Bruchsal, pour sa part, est aujourd'hui malheureusement perdu, et sa disparition inexpliquée a dû intervenir entre 1438 et 1466 puisqu'il n'apparaît plus dans l'inventaire des livres conservés à l'église du Saint-Esprit établi à cette date ${ }^{31}$.

Ludwig Schuba a en outre repéré le nom de Hildegarde au f. $65 \mathrm{v}$ du ms. Pal. lat. 1207, exécuté par Gerhard von Hohenkirchen (vers 1382-1448) : maître ès arts et docteur en médecine, il s'était établi à Heidelberg en 1420, où il fut nommé recteur de l'université en 1429, après l'avoir été de celle de Cologne en $1418^{32}$. Outre la liste des recteurs de l'université de Cologne entre 1389 et 1418, ce manuscrit contient les statuts que l'université de Heidelberg s'était donnés en 1425, apparemment inspirés de ceux de la faculté de médecine de Cologne, fondée en $1313^{33}$, et différents traités médicaux de Gerhard von Hohenkirchen : entre autres, un long poème didactique en hexamètres, Metrum de accidenti et morbo Galeni.(ff. 18r-59r) et .un traité De febribus (ff. 60r-91r) d'après différentes autorités médicales, de l'Antiquité mais aussi du Moyen Age ("Gilbertus" f. 85v, "Johannes de Sancto Amando" et "Constantinus" f. 87v, "Platearius" f. 89r, etc..). Le folio $65 \mathrm{v}$ contient ainsi de nombreux extraits mis bout à bout de la Physica de Hildegarde, mais c'est en

\footnotetext{
${ }^{29}$ Cf. Bibliotheca Palatina, Katalog zur Ausstellung vom 8. Juli bis 2. November 1986 Heiliggeistkirche Heidelberg (désormais cité Bibliotheca

Palatina), Textband herausgegeben von Elmar Mittler in Zusammenarbeit mit Walter Berschin, Jürgen Miethke, Gottfried Seebaß, Vera Trost, Wilfrid Werner, Heidelberg, Braus, s. d., pp. 7-8.

${ }^{30} \mathrm{Ce}$ manuscrit, édité par Paul Kaiser au début de ce siècle, se trouve aujourd'hui à Copenhague (Kongelige Bibliothek, Ny. kgl. saml. 90b).

${ }^{31} \mathrm{Cf}$. L. Schuba, Die medizinischen Handschriften der Codices Palatini Latini in der Vatikanischen Bibliothek, op. cit., pp. XXVII-XXVIII.

${ }^{32}$ Sur Gerhard von Hohenkirchen, voir G. Keil, Die deutsche Literatur des Mittelalters Verfasser Lexikon, Band 4, "Lieferung 1", neu arbeitete Auflage, Berlin/ New York, De Gruyter, 1982, col. 99. II mourut en 1448, léguant au "Collegium Dionysianum" de l'Université de Heidelberg son importante bibliothèque personnelle d'après Bernd Lorenz, "Notizen zu Privatbibliotheken deutscher Ärzte des 15.-17. Jahrhunderts", Sudhoffs Archiv, 67, 1983, Heft 2, pp. 190-198.

${ }^{33}$ Cf. Bibliotheca Palatina, op. cit., p. 63.
} 
fait également un fragment de cette œuvre qui se cache derrière la rubrique "Empirica" au f. 64r ${ }^{34}$.

Je compte donner dans ma thèse une édition de ces deux fragments, précieux pour l'histoire de la circulation et de la réception de l'œuvre scientifique de Hildegarde à la fin du Moyen Age. Ils suggèrent en effet que non seulement l'actuel Causae et curae mais aussi la Physica étaient connus et présents au XVe siècle dans les milieux liés à la faculté de médecine de Heidelberg; mais surtout ils attestent que l'œuvre médicale de Hildegarde, citée par un médecin à l'égal d'Albert le Grand ou Arnaud de Villeneuve, faisait autorité en la matière, à ses yeux, au même titre qu'eux.

Cette dimension sociologique et géographique de sa réception est confirmée par notre second fragment anonyme, qui contitue un autre exemple de l'utilisation de la Physica par un médecin enseignant à l'université de Heidelberg au XVe siècle.

\section{Le manuscrit Pal. lat. 1144 (Cf. Annexe B)}

Le ms. Pal.. lat. 1144 est lui aussi un recueil empruntant à de nombreux auteurs scientifiques (Isidore de Séville, Arnaud de Villeneuve, le PseudoAlbert, Thomas de Cantimpré, Rhazès, etc.). Il se compose de 316 folios de 20, $5 \times 15,5 \mathrm{~cm}$ et comprend deux parties : la première (ff. 1-296, papier) date de la deuxième moitié du XVe siècle et est originaire de Heidelberg ; elle rassemble des cahiers de différentes origines, copiés par plusieurs mains. La seconde partie en revanche (ff. 297 à 316) est constituée de deux quinions de parchemin et remonte au XIVe siècle ; elle est due à une seule main, qui y a copié des textes philosophiques ${ }^{35}$.

Ce manuscrit appartenait au XVe siècle à Erhard Knab, personnage bien connu grâce aux travaux de Colette Jeudy et Ludwig Schuba. Né en 1420 à Zwiefalten, en Souabe, il se transféra à Heidelberg où il fut immatriculé en mai 1439. Promu au rang de baccalarius artium l'année suivante, puis à celui de

34"Empirica" est ici écrit à l'encre rouge, comme le nom des œuvres ou des auteurs cités dans ce manuscrit entre les folios 60r et 91r (65v "Gilbertus", 85v "Albertus in de natura rerum", 89r "Thesaurus pauperum", etc.); en fait, le nom d'Hildegarde apparaît juste après cette rubrique : Hildegardis dicit contra febres ardentes pulverisa galangam ...

${ }^{35}$ Albertus Magnus, de impressionibus aeris ff. 297r-303v, Notabilia logicometaphysica ff. 303v-304v, Johannes Dacus, Divisio scientiae seu communia ad omnem materiam ff. 304v-311v, de vita Aristotelis ff. 311v-313r, liber de pomo sive de morte Aristotelis ff. 313-316v. 
licentiatus artium en 1443, il fut élu doyen de la faculté des arts en 1451 et commença alors des études de médecine. Baccalarius medicinae en 1453, recteur de la faculté des arts en 1455, il est mentionné pour la première fois en tant que licentiatus medicinae en 1462 sans qu'on connaisse la date exacte de sa promotion, assurément postérieure à 1456 si l'on en croit le ms. Pal.. lat. 1083, où il se présente ainsi, f. 40vb :

Scriptus ...et lectus est ille liber ...per me Erhardum Knab arcium magistrum et medicine baccalaureum in universitate Haidelbergensi anno domini 1456 die 25 Februarii.

Autorisé à s'absenter pendant un an et à "pratiquer" à Spire en 1464, il fut élu recteur de l'université de Heidelberg en 1465 (il le sera à nouveau en 1470 et 1476), et participa à ce titre à l'élaboration du catalogue des livres de I'Université de Heidelberg en 1466. II mourut en 1480, au terme de plus de trente ans d'activité académique, laissant une œuvre très variée : différents discours, de nombreux commentaires (sur les Summulae logicales de Petrus Hispanus, la Rhétorique d'Aristote ou l'Ars minor de Donat, mais aussi sur Galien, Hippocrate, Avicenne, Gilles de Corbeil, etc)., un Aggregatorium Rhetoricae qui constitue son œuvre la plus accomplie en tant que magister artium, et des écrits pratiques. Parmi les œuvres qu'il composa pendant son activité d'ordinarius de médecine on compte des Consilia medica et des Regimina (entre autres pour l'évêque de Spire ${ }^{36}$ ) et surtout une Practica tam in phisicis quam in chirurgis composée entre 1464 et 1466, en deux parties : la première s'intitule Practica medicinalis ou Viaticus en référence à Constantin I'Africain, la seconde, Chirurgia bona. Toutes deux ont été compilées par ses soins pour servir de manuel aux praticiens. "In unum collectus pro utilitate sua propria et aliorum practicare volentium", ainsi décrit-il son Viaticus, tiré des écrits d'Arnaud de Vileneuve, de Guillaume de Saliceto, etc ${ }^{37}$.

On connaît actuellement une vingtaine de manuscrits ayant appartenu à Erhard Knab, et il s'agit, pour la plupart, de manuscrits de médecine écrits soit par lui soit pour lui. Le ms. Pal.. lat.1144 contient ainsi des textes que Knab a sans doute achetés non reliés, pour les faire relier plus tard avec les textes de sa main (souvent intercalés dans d'autres œuvres) dont on trouve des

\footnotetext{
${ }^{36} \mathrm{Cf}$. L. Schuba, Die medizinischen Handschriften der Codices Palatini Latini in der Vatikanischen Bibliothek, op. cit., p. XXXIII.

${ }^{37}$ La plupart de ces renseignements sont tirés de l'article de Colette Jeudy et Ludwig Schuba, "Erhard Knab und die Heidelberger Universität im Spiegel von Handschriften und Akteneinträgen", Quellen und Forschungen aus italienischen Archiven und Bibliotheken, 61, 1981, pp. 60-108. D'autres proviennent de Bibliotheca Palatina, op. cit., pp. 14, 22-23, 64.
} 
exemples aux folios 31rv, 48rv, 133rv, 166r-188v, 191r, 192v, 193v-200v, $201 v-215 v$ ou $240 r-244 r$.

Il a copié également les folios $128 \mathrm{v}$-129r, qui suivent dans le recueil les Tabulae salernitanae sive compendium magistri Salerni cum apparatu Johannis de Sancto Paulo (ff. 86r-127v). Le folio 128r est vierge, les folios $129 \mathrm{v}-132$ le sont également ; le texte est écrit sur une seule colonne à l'encre noire, sans rubrication, et comprend 31 lignes au f. 128v et 20 lignes au f. 129r.

Six chapitres du "livre des plantes" sont ici représentés, qui s'ouvrent tous, comme dans la Physica, sur le nom de la plante qui fournit leur tête d'article (à l'exception du dernier paragraphe de notre texte qui indique une des applications médicales possibles du "champignon qui pousse sur le poirier" avant de le nommer [B6] ) et dans la plupart des cas sur l'énoncé de leur complexion : la Fougère est "chaude et sèche" (B1), la Morelle également (B4), I'Aigremoine est chaude (B3), tandis que le Calendula apparaît comme une plante "froide et humide" (B5). Dans l'ordre, on lit donc dans ce fragment des extraits des chapitres suivants du livre premier : 47, "De Farn", 57, "De Winda", 114, "De Agrimonia", 121, "De Nachtschade", 122, "De Ringula", et 172 "De Fungis".

La succession des extraits reflète l'ordre de la lecture, de même qu'est respecté ici l'ordre des phrases de chaque chapitre utilisé : le travail de Knab se réduit à une entreprise d'extraction au fil du texte original, sans réorganisation. Les maladies évoquées dans ces lignes sont de ce fait éparpillées, et très diverses : on y trouve les affections oculaires (B1c et B3c), la surdité (B1d), le mal de dents (B4b), la faiblesse cardiaque (B4a) ou cérébrale (B5a), les pieds gonflés (B4C), etc., et même les maladies du bétail (B5b et $C$ ) ; mais on voit aussi pris en compte la condition de parturiente (B1a), la magie (B1a et g) ou l'empoisonnement (B1g et B5a), ou encore le soin des ongles - en cas de gale ?- (B2 et B6). Soulignons au passage que la première des deux recettes ad unguium deformitatem (B2) constituait, à l'époque de Hildegarde, l'une des premières mentions de l'usage thérapeutique du mercure ("vif argent"), en l'occurrence dans le domaine dermatologique : un emploi similaire est prôné dans un autre écrit attribué à l'abbesse, le "Fragment de Berlin"38, et il est probable, pour Irmgard Müller, que Hildegarde ait eu connaissance de cette

${ }^{38} \mathrm{Cf}$. Heinrich Schipperges, "Ein unveröffentlichtes Hildegard-Fragment (Codex Berolin. Lat. Qu. 674)", Sudhoffs Archiv für Geschichte der Medizin, 40, 1956, pp. 41-77, p. 53 : Tunc ille sulphur et vivens argentum et hircinum sepum ac butyrum accipiat et unguentum inde faciat... 
utilisation possible du mercure par la lecture de Constantin l'Africain (†1087) ou d'autres écrits de médecins salernitains ${ }^{39}$. '

De même que dans notre premier fragment, les simples énumérés par Knab ne sont en effet pas tous utilisés seuls : outre le "vif argent" associé au liseron, on trouve ici le vin utilisé comme excipient dans une médication à base d'aigremoine contre l'excès de flegme (B3a) ou dans un antidote dont le calendula est la principale composante (B5a), mais aussi le lard qui, écrasé avec cette même plante, permet de préparer un onguent contre la teigne (B5d) ou encore le fiel de bœuf, dans lequel il convient de tremper "le champignon qui pousse sur le poirier" (B6) pour soigner les ongles - le principal facteur de réussite de cette dernière recette ne résidant d'ailleurs pas tant dans la nature des ingrédients que dans la taille du champignon : c'est la similitude des formes qui permet ici de soigner un ongle malade avec un champignon de même taille ; de même, dans une autre recette de la Physica, des morceaux de laitue de même dimension que les scrofules sur lesquelles on les applique, peuvent en venir à bout 40 .

Les recettes sélectionnées par Erhard Knab se distinguent donc par une plus grande fidélité à leur source (on n'y relève guère comme variante que "novem diebus et noctibus...in felle intingat" [A26] qui ne figure pas dans le chapitre reproduit par Migne ${ }^{41}$ ) ainsi qu'une plus grande uniformité linguistique. Les plantes sont présentées sous une appellation unique, latine dès que cela est possible si l'on excepte le Liseron et le Calendula, cités faute de mieux sous leur nom germanique ("Winda" [B2], "Ringula" [B5]). Mais il n'est guère possible d'aller au-delà de ce constat, et de savoir par exemple si la faible proportion d'emprunts à la langue vulgaire reflète la bonne connaissance qu'avait Erhard Knab de la langue latine, ou la leçon du manuscrit qu'il compilait.

Le manuscrit Pal. lat. 1144 nous fait donc connaître un médecin de plus parmi les utilisateurs de l'œuvre scientifique de Hildegarde au Moyen Age, outre le "Doctor Barbatus" qui posséda le manuscrit 525 de Berne d'après l'exlibris qui y figure ${ }^{42}$, mais aussi le "cyrurgus" Guillelmus qui, au XIVe siècle,

${ }^{39} \mathrm{Cf}$. Irmgard Müller, "Krankheit und Heilmittel im Werk Hildegards von Bingen", dans Hildegard von Bingen 1179-1979, Festchrift zum 800. Todestag, hrsg.

Anton Ph. Brück, Mayence, 1979, pp. 311-349, p. 327.

${ }^{40}$ Cf. Physica, I, 9, "De lactuca agresti", PL 197, c. 1165C : Si quis etiam scrofulas habet, antequam rumpantur, accipiat lactucam...et iuxta eamdem abrumpat secundum latitudinem scrofularum...

${ }^{41}$ Cf. Physica, I, 172, PL 197, c. 1195D.

42"Doctori Barbato pertinet"; on ignore tout de ce personnage, mais on sait qu'un autre manuscrit conservé à Berne (Cod. 227) était également sa propriété 
acheta l'actuel ms. Ashb.1323 au "cyrurgus" Guido pour 6 florins, comme

l'atteste une inscription sur le dernier folio de ce manuscrit, ou encore Gerhard von Hohenkirchen. Dans une perspective pllus large, ce fragment nous livre aussi un témoignage supplémentaire sur la spécificité de l'enseignement dispensé, à la fin du Moyen Age, à la toute nouvelle faculté de médecine de Heidelberg.

On sait par exemple qu'Erhard Knab s'est intéressé à un auteur alors un peu oublié, Gilles de Corbeil, et son commentaire du De urinis de ce médecin du XIIle siècle constitue au XVe siècle, selon Danielle Jacquart et Françoise Micheau, une "résurgence un peu archaïque"43. Or c'est peut-être l'enseignement même de la médecine à Heidelberg à cette époque qui présentait des traits archaïsants : il s'avère que non seulement le traité des urines mais aussi le poème médical sur le pouls, De pulsibus, de ce même Gilles de Corbeil, faisaient partie des œuvres que les bacheliers désireux d'obtenir la licence étaient tenus de lire. Les statuts de la faculté de Heidelberg copiés par Gerhard von Hohenkirchen dans le ms. Pal. lat. 1207 contiennent en effet un chapitre "de licendiandis" (f. 5 r) où sont énumérées ces œuvres : outre Gilles de Corbeil (baccalaurus volens promoveri ad licenciam teneatur legere continue et per duos annos ad minus...versus Egidii de urinis et pulsibus cum suis commentis pro uno cursu) on y trouve une traduction de Constantin I'Africain, le Pantegni (libri Tegni Galieno cum commento Hali pro uno cursu), pourtant "de moins en moins utilisé", toujours selon D. Jacquart et F. Micheau, "à partir du XIIle siècle"44. II est probable en tout cas que l'intérêt d'un Gerhard von Hohenkirchen et d'un Erhard Knab pour la Physica de Hildegarde, produit de la médecine du cloître du XIle siècle, aille plus généralement de pair avec l'importance qu'avait toujours la médecine salernitaine, y compris ses auteurs les plus archaïques ${ }^{45}$, dans l'enseignement médical à Heidelberg au XVe siècle.: leur utilisation d'un auteur comme Hildegarde, délaissé, voire ignoré, dans les autres milieux universitaires européens à la même époque, est donc

en 1472 (cf. Lynn Thorndike, "Some later medieval latin medical manuscripts at Bern and Prag", Annals of Medical History, 1936, new series, vol. 8, pp. 427432, p. 428.)

${ }^{43}$ Cf. D. Jacquart, F. Micheau, La médecine arabe et l'Occident médiéval, Paris, Maisonneuve et Larose, 1990, p. 196.

${ }^{44}$ Ibidem, p. 174.

${ }^{45}$ Gerhard von Hohenkirchen cite par exemple Gariopontus (†1050), f. 93v (Item Gariupoton salernitanus in capitulo de curatione febris singularis scribit sic ) et assimile par ailleurs Gilles de Corbeil à l'Ecole de Salerne, f. 144r : Egidius Salernitanus in prologo suo super metrum quod ipse fecit de judiciis urinarum... 
un indice supplémentaire de la diffusion de la Physica dans l'aire germanique, tout autant qu'un signe de la jeunesse de la faculté de médecine de Heidelberg.

Nos deux nouveaux fragments anonymes permettent donc de préciser la géographie de la circulation des écrits scientifiques de Hildegarde, manifestement concentrée le long de l'axe rhénan, aux derniers siècles du Moyen Age. Mais les différences qui les opposent nous renseignent également sur les transformations qu'a pu subir la Physica entre l'époque de sa composition et le XVe siècle : plus encore que les préoccupations divergentes de leurs copistes respectifs, les mss Pal. lat. 1216 et 1144 reflètent sans doute un état différent des sources, directes ou non (l'anonymat de ces fragments pouvant également être dû à une connaissance indirecte de l'œuvre). Plusieurs versions de la Physica coexistent en effet à cette époque, intégrales ou morcelées. Erhard Knab, copiste du fragment contenu dans le ms. Pal. lat. 1144, semble ainsi n'avoir disposé que de son livre premier, contrairement au scribe de notre second fragment, et le $\mathrm{ms}$. Pal. lat. 1207 suggère une hypothèse semblable : le folio $64 \mathrm{r}$ n'y est constitué que d'extraits du "livre des plantes" de Hildegarde, tandis que le f. 65v emprunte à ce même livre, mais aussi aux livres III ("des arbres"), IV ("des pierres") et VII ("des animaux") de la Physica. II est donc possible que le "livre des plantes" ait été détaché des autres à une date inconnue, pour former un herbier autonome, circulant parallèlement à une Physica complète. Ces deux nouveaux fragments témoignent donc de l'intérêt suscité encore au XVe siècle en Allemagne, notamment à Heidelberg, par l'œuvre médicale d'une abbesse du XIle siècle, mais aussi des avatars subis par cette œuvre en l'espace de trois siècles, prix probable de sa transmission hors du cloître où elle avait vu le jour. 


\section{Annexe A}

Manuscrit Pal. lat. 1216, transcription des folios 91v-95r:

Nous avons respecté l'orthographe et la mise en page (alinéas) du fragment; seules une ponctuation minimale et une numérotation des recettes ont été introduites.

(f. 91 v) [A1] Si boves aut oves aliquid mali comederint unde repente inflantur, herba quae dicitur Ryngele sive flammula tundatur, et sucus ejus exprimatur, et cum modica aqua idem sucus eiusdem herbe in ora eorum fundatur ut gustent, et curabuntur.

[A2] Si autem bovis aut ovis tussitat, sucus eiusdem herbe naribus eorum infundatur, et humores noxios excreant et viribus eiusdem herbe meliorabuntur.

[A3] Si mortalitas pecora occidit, corticem arboris castanei contunde ponens in aquam ut inde saporem habeat, et de hac aqua sepe potabis ea ; tam equis asinis bobus ovibus porcis quam ceteris pecoribus prodest et curabuntur. [A4] Quod si etiam bos, equus vel asinus pinguescere incipit eo quod vel potum vel pabulum nimis avide sumpsit, quod vulgariter dicitur virfangen vel zerese, da illi folia eiusdem arboris in pabulo ad comedendum si potest, sed si comedere noluerit ipsa folia pulveriza in aqua da bibere pulveres.

[A5] Si oves infirmari incipiant, feniculum et parum minus de aneto in aquam pone ut saporem ex eis concipiant, et infirmis ad potandum da et sanabuntur.

[A6] Si reuma equo per nares fluit ita quod inde tussiat, libisticum et parum minus urentis urtice simul in aqua coque, deinde herbis ablatis fumum earumdem herbarum in nares eorum et os [per]mitte intrare, freno in os posito vel ligno, ut fumus in os et frontes transeat et in caput, et curabitur.

[A7] Si autem equus in ventre dolet velut de morsibus fiat easdem herbas pabulo eius saepe intermisce ut urticam et pabulum et libisticum simul sumat, et curabitur.

[A8] Si equi aut asini boves oves capre porci canes alia quaecumque animalia constrictionem guthuris id est strengelen patiuntur, seu dolorem capitis id est heubtsucht habent, magnam massam cupri in caldarium vel ollam vel patellam pone fundens aqua desuper, fortiter fac bullire ac deinde cum aqua fervente eadem pastum eorundem iumentorum, sive (f. 92r) avena sit sive fenum, semel vel bis asperge, et comedit et dicte pestes cessabunt.

[A9] $\mathrm{Si}$ canis insanit caput laudule ascide et da ut comedat, et furorem dimittit et mansuetus fit. 
[A10] Si canis aliquem momorderit, de farina simile et albugine ovi pastam faciat et eam super morsum canis ponat per tres dies et noctes ut eundem venenosum morsum extrahat, et post hec pasta ablata millefolium cum albugine ovi contundat et super eundem morsum per duos vel tres dies ponat ; post hec etiam hiis ablatis ipsam plagam sanat ungentum ut aliud vulnus sanari solet.

[A11] Contra bubonem struma pavonis desicca et reserva, et si ulcus vel bubo id est sler ebulit eam desuper liga, et suaviter rumpetur ; et cum ruptum fuerit iterum desuper pone, putredinem extrahit et curat.

[A12] Si pustula surgens in nigredinem vertitur in qua mors hominis est, folia vel radix herbe que dicitur aaron id est jarus vel serpentina comedatur et si in ipsa pustula nimis ardor sit vel nimium frigus, utraque mitigat ita quod ipsa pustula suaviter deficit.

[A13] Contra ortorum vermes terram quae sub arbore est quae dicitur esculus id est spirbaum et circa radices eius in ortos sparge ubi eruce id est rupen et papiliones id est cyfelder olera comedunt et devastant ; omnes recedunt vel moriuntur.

[A14] Contra vermes hominis vel pecudis accipe ossa lucii et pulveriza et pulverem super locum doloris pone et vermis moritur.

[A15] Ad muscas necandas recipe zizaniam id est rade et mel commisce, et pulverem piretri et cuperrose id est nesewurtz et cuperrauch simul commisce ; postea pulverem calido vino adjunge et parietibus hec illinias et quaecumque (f. 92v) musce inde gustaverint infirmabuntur et deficient et cadent et moriuntur ; si mel non habes commisce cum lacte quocumque.

[A16] Contra surditatem, si humoris fumositas caput fatigat ita quod aures tinniunt velut sonitus aquarum et quod inde auditus impeditur, herba que dicitur gunderabe in aqua ferveri faciat et expressa aqua ita calidam capiti circumponat et tinnitus minuitur et auditus aurium aperitur.

[A17] Qui surdus est ita quod non audit, lac femine que masculum peperit cum iam duodecim septimane sunt quod filium genuit, et de suco herbe que dicitur semperviva id est huswurtz illi lacte addat, et in aurem dolentis tepidas tres guttas aut quatuor modice instillet et hoc si sepe facit [manque]

[A18] Contra vocis raucetudinem ex causa humida vel ex asmate, liquiricium et radicem pyonie equaliter pulveriza et de pulvere herbe que brachwurtz dicitur, tertium quantum istorum duorum est ; simul commisce, modico lucido sale addito vel asso sale, et hunc pulverem tam ieiunus quam pransus sepe comedat ; vocem serenam facit, pectus sanat, paralisim deprimit. [A19] Qui in stomacho dolet, zinziber et cedoar equaliter et galange quantum istorum duorum est pulveriset, et hunc pulverem calido vino inponat et post 
cibum bibat ; et similiter ad noctem cum dormitum vadat et hoc sepe faciat et sanus erit.

[A20] Cui cerebrum infirmum est et velut vacuum serpillum pulverizet et hunc pulverem cum farina simile commisceat in aqua et tortellas de hac pasta pistas sepe comedat et cerebrum confortatur. [A21] Item scarleia cocta et calida capiti imposita idem facit per noctem.

[A22] Contra oculorum ulcerationem, zinziber pulverizatum in pannum liga et vino inpone ut vinum ex hoc acerbum fiat, et cum vadis dormitum eodem vino palpebra circumlinias et oculos, et si intrat oculos non nocet.

(f. 93r) [A23] Item herba pentafilon funfblat in purum vinum pone, et cum ipso in mortario contunde et sic per pannum cola et idem vinum in ereo vaso reserva ; et cum dormitum vadat, de ipso vino cum penna circa oculos lineas ita quod etiam modice oculos intret.

[A24] Qui in dentibus dolet, solatrum id est nachtschade in aqua coquat et eam cum dormitum vadat super maxillam ubi dolet superpone calidum, et valet.

[A25] Qui in epato vel pulmone et etiam in visceribus dolet linguam cervinam in vino fortiter coquat et vino cocto purum mel addat, et iterum semel fervere faciat et deinde per pannum colet, et hoc claretum tam ieiunus quam pransus bibat ; epati prodest, pulmonem purgat, dolentia viscera sanat, interiores putredines aufert.

[A26] Qui tussi sicca gravatur cum tanaceto, id est reynevane, et farina similigena sorbitium faciat et sepe comedat ; et ariditas ac interiora ulcera eiusdem tussis solventur et excreantur per os.

[A27] Contra frenesim et renum aut dorsi dolorem vulturem occide et depluma et exentera quem frustratim incide, et in nova olla cum aqua decoque diu et pinguedinem super natantes diligenter collige aliis carnibus abjectis ; huic sagimini parum minus olei olive adde et minus olei iusquiami ; hoc ungentum in vitreo vase reserva et frenetici caput totum rasis crinibus inunge aut si renibus aut dorso patitur ibidem eum inunge, et curabitur nisi deus nolit quia ungentum istud efficacissimum est et cito cutem penetrat.

[A28] Qui in corde defectum patitur vel qui tristatur, solatrum in aqua coquat et expressa aqua calidam herbam super cor ponat. [A29]Item herbam wullene, cum carnibus vel piscibus coquat vel in tortellis sine aliis herbis, et sepe comedat (f. 93v); cor inde confortatur et letum erit.

[A30] Qui debile cor et stomachum frigidum habet, herbam que dicitur peffircrut crudam comedat et eum confortat et letificat, oculos clarificat nam sanis et infirmis comesta utilis est et bona, et acerbitas saporis interiora hominis non mordet sed sanat. 
[A31] Ungentum quod hylarius egiptius docuit contra dolorem utriusque lateris et pectoris quocumque modo quis doleat et contra paralisim. Recipe folia persici voluntate et tantum sysemare crut, et ad eius tertiam partem basilie, ad pondus basilie plantaginem ; hec diu in aqua coque, per pannum cola et fortiter contorque ; post hec oleum laurinum at bis tantum sepi cervini vel yrcini et ad eius tertiam partem veterem arvinam cum praedicta aqua in patella super ignem liquefac et commisce, et cum hec omnia bene incorporata fuerint infrigidari permitte atque hoc ungento latus vel pectus vel ubi paralisis fuerit te perunge, et curaberis.

[A32] Pulvis bonus Recipe semen feniculi quantumvis, dimidiam partem galange, ad medietatem galange dyptamnum, ad eius medietatem pilosellam ; hec simul pulveriza et combura atque post modicam horam prandii in vino calido bibe ; hic pulvis si sanus es te conservat sanum, infirmum confortat, bonam digestionem facit et vires tribuit, bonum et pulchrem colorem facit sanis et infirmis, post cibum comesta conveniens est et bona.

[A33] Pulvis optimus. Recipe radicem et folia herbe que dicitur (f. 94r) Rustica id est biverwurtz, pulveriza, et ad eius medietatem piretrum, ad piretri medietatem cynamomum ; hos pulveres simul commisce, comede cottidie vel cum pane vel in sorbitio vel vino calido sume, et nullam magnam vel diuturnam egritudinem habebis ; et nullus hoc pulvere careat quia sanus si hunc pulverem comedat infirmus ad longum tempus non erit ita ut in lecto decumbat, et si eo infirmus utatur cotidie, sanus erit ; si hunc pulverem per annum servare vis in victili vase sub terra fodias.

[A34] Electuarium de piris Recipe pira bona, incide, et reiecta interiora duritia et nucleis ipsas fortiter in aqua coque et in mortario contunde ut fiant quasi pasta ; postea herba que dicitur berwurtz id est peucedanum et si peucedanum non habes accipe radices feniculi pro medietate pirorum, et galange parum minus, et liquiricii minus quam galange et haec pulveriza et cum melle despumato leniter coque et commisce ut inspissetur ; et tunc predicta pira adde et hec omnia commisce fortiter cum cocleari circummovendo atque vasi reserva ; et hoc cottidie mane comede ad medie nucis quantitatem et post prandium ad nucis integre magne quantitatem de vespere ad quantitatem nucis et dimidietate ; hoc electuarium pretiosius et utilius est auro quia emigraneam auffert et gravedinem pectoris quam cruda pira generant scilicet asma minuunt, et omnes malos humores in homine consumit, et sic purgat homines velut vas a fece purgatur.et corpus conservat sanum.

[A35] Qui febres quartanas patitur, fenum grecum (f. 94v) in aqua coquat et expressa aqua illud utrisque pedibus et tibiis calidum circumponat et ligaturis 
constringat ; hoc sepe faciat et etiam fenum grecum in vino calefaciat et bibat calidum et curabitur.

[A36] Contra tertianas bibe sucum plantaginis mane per tres [dies] et curaberis. [A37] Contra cottidianas, ligaturam ex panno canapino fac, cui aloe super illinias, quod stomacho et umbilico superponas, febres omnes hoc modo fugat.

Cum non sit dubium medicinalem virtutem inesse lapidibus pretiosis de quibusdam virtutes scribere jam propono de iacincto et onicho de saphiro de topazio.

[A38] Si vermis homines comedat lineum pannum super plagam pone et desuper smaragdum, et super ipsum alios panniculos pone ligando tanquam sit cauterium, ut idem lapis ibidem incalescat, et sic per tres vel plures dies faciet et vermis morietur.

[A39] Qui caliginem oculorum patitur vel ulcerationes, jacinctum ad solem teneat ; et cum incaluerit, statim saliva eum madefaciat, sic oculis citissime apponat ut inde calefiant ; hoc saepe faciens oculi clarificantur.

[A40] Item vinum bonum et clarum mitte in vasculum eneum vel cupreum vel calibineum, et onichinum inpone ut in eo per $X X$ vel $X X X$ dies iaceat, et tunc ablato lapide idem vinum in vasculo reserva et singulis noctibus eodem vino oculos circumlinias ita ut oculos modice intus tangat; valet.

(f. 95r) [A41] Cui pellicula in oculo crescit, saphirum in manu sine igne calefaciat, et postea ipsum in gutta boni vini madefaciat et pelliculam in oculo cum saphiro sic madido tangat per tres dies vel plures mane et vespere ; pellicula minorabitur et evanescit praeterea cui pre dolore rubent vel caligant: saphirum ieiunus in os ponat et sic humectatum oculis circumliniat ut interius oculos tangat.

[A42] Item topazion per tres dies et noctes in clarum et bonum vinum pone et cum dormitum vadis, cum eodem lapide sic madido oculos circumlinias et ut idem liquor interius oculos tangat; et topazio ablato vinum hoc per $\mathrm{V}$ dies servare potes sed quotiens oculos ad noctem linire volueris eundem lapidem in vino prefato intingas et sic madido oculos circumlinias et hoc saepe fac, semper post $\mathrm{V}$ dies vinum cum topazio renovando ; et hoc oculos velut optimum colirium clarificat et conservat. 
Manuscrit Pal. lat. 1144, transcription des folios 128v-129r:

Nous avons numéroté de 1 à 6 les passages empruntés à autant de chapitres de la Physica ; le cas échéant, plusieurs recettes tirées d'un même chapitre ont été distinguées par des lettres.

[B1](f. 128v) [a] Filix calida et sicca valde hominem preservat ab incantacione et magica illusione ideo quod valet pro parturientibus quibus et pueris natis circumponatur. [b] Contra artheticam ex frigida causa valet si quis balneet ex ea. [c] Aufert caliginem oculorum si superponatur et homo sic dormiat sepe et hoc cum viridis est. [d] Semen eius in panniculo ligatum et in aure positum in panno ne intus cadat auditum perditum reformat. [e] Semen ejus super linguam positum valet sue paralisi. [f] Semen in manu tentum ut calefiat facit redire ad memoriam et facit hominem bone inteligentie. [g] Item ubi crescit aut in domo est valet contra venena et magica et fantasias et raro ibi cadunt grandines fulmina et huiusmodi.

[B2] Winda tundatur et addatur modico argenti vivi et super ungues ponatur et panno ligetur et pulchri erunt.

[B3] [a] Agrimonia calida est et in quo scientia et sensus evacuati sunt illi crines abradantur et agrimonia in aqua coquatur de qua caput lavetur et eadem herba calida super cor ejus ligetur panno quam primum cordis defectum sentiet et etiam ponatur calida super frontem et timpora. [b] Qui etiam flecma de infirmis visceribus excreat et frigidum stomachum habet agrimoniam in vino bibat et excreationem minuit et stomachum confortat.

[c] Item contundetur in mortario et trita super oculos ad noctem ponatur et ita pannum desuper liga cavens ne oculos intret caliginem tollit et claritatem inducit.

[B4] Solatrum calidus et siccus [a] Qui in corde dolet aut impotentiam habet coquet ipsum in aqua et aqua expressa eam calidam super cor ponat.

[b] Item qui dolet dentes calefaciat ipsum in aqua et cum dormitum vadit super maxillam ponat ubi dolet .

[c] Item cui pedes tument eam in aqua modice calefactam pedibus superponat. [d] Item qui in medulla in cruribus dolet herbam calefactam cruribus circumponat et panno circumliget.

(f.129r) [B5] Ringula frigida et humida et fortiorem viriditatem in se habet. [a] Qui venenum comedit eam in aqua coquat et aqua expressa eam super stomachum ponat et venenum mollificat ut exeat sed et idem bonum vinum mox calefaciat et de ea inponat et semi calidum illud vinum bibat et venenum aut per 
nasum spumando eicit aut per aliam spumam. [b] Contra tumorem repentinum pecorum tundatur et succus eius in os fundatur ut inde gustent et curabuntur cum aqua modica. [c] Contra tussim pecorum succum illum naribus sine aqua infunde. [d] Contra ulcerationem capitis capiat durum circa lardum et cum ea in mortario tundat et caput ungat. [e] Contra scabiem capitis recipe fol[ia] et flores eius et succum exprime et de succo isto et modica aqua et cum farina tritici vel siliginis pastam faciet et cum illo totum caput suum panno cum pileo ligatum relinquat dum illud incalescat et rimas pasta habeat ; tunc deponat et iterum renovet per novem dies Et quotiens aufert lixivio parato cum eius succo caput abluet.

[B6] Ad unguium deformitatem Accipe fungum piri ad latitudinem digiti sive unguis et in felle bovis viri intingi et ita partem illam in felle intinctam ungui superliget novem diebus et noctibus denuo cum exiccatur iterum in felle intingatur. 
Annexe C

Table de concordances

Ms. Pal. lat. 1216

rang de la recette

chapitre correspondant dans la Physica éditée par

Migne

01

02

03

04

05

06

07

08

09

10

11

12

13

14

15

16

17

18

19

20

21

22

23

24

25

26

27

28

29

30

31

32

1158A
I, 122 "De Ringula", c. $1179 \mathrm{C}$

I, 122 "De Ringula", c. 1179D

III, 12 "De Kestenbaum", c. 1226C

III, 12 "De Kestenbaum", c. 1226C

I, 66 "De Feniculo", c. 1158B

I, 100 "De Urtica", c. 1169B

I, 139 "De Lubestuckel", c. 1186C

IX, 5 "De Cupro", c. 1349B

VI, 45 "De Lercha", c. 1304C

I, 1 "De Tritico", c. 1129C

VI, 3 "De Pavone", c. 1289A

I, 49 "De Herba Aaron", c. 1149A

III, 8 "De Spirbaum", c. 1225A

V, 9 "De Hecht", c. 1276B

I, 12 "De Ratde", c. 1133D

I, 105 "De Gunderebe", c. 1171A

I, 42 "De Huszwurtz", c. 1145D

I, 54 "De Brachwurtz", c. 1150B

I, 15 "De Ingeber", c. $1136 \mathrm{C}$

I, 32 "De Quenula", c. 1142D

I, 161 "De Scharleya", c. 1191C

I, 15 "De Ingeber", c. 1136A

I, 55 "De Funffblat", c. 1150D

I, 121 "De Nachtschade", c. 1179B

I, 30 "De Hirtzunge", c. 1142A

I, 111 "De Reynfan", c. 1173D

VI, 7 "De Vulture", c. 1291B

I, 121 "De Nachtschade", c. 1179B

I, 123 "De Wullena", c. 1180B

I, 38 "De Pefferkrut", c. 1144B

III, 58 "De unguento Hilarii", C.1246A

I, 66 "De Feniculo", c.

I, 146 "De Byverwurtz", c. 1188D

III, 2 "De Birbaum", c. 1218D

I, 36 "De Fenugreco", c. 1143D

I, 101 "De Plantagine", c. 1169B ? 
IV, 1 "De Smaragdo", c. 1250C

IV, 2 "De Jacincto", c. 1251A

40

IV, 3 "De Onichino", c. 1251D

IV, 6 "De Saphiro", c. 1253BC

IV, 8 "De Topazio", c. 1255CD 